\title{
COMPORTAMIENTO DE GENOTIPOS DE PAPA EN LA ZONA CENTRAL Y SUR DE CHILE
}

\author{
CLAUDIO R. SANDOVAL B.; JOSÉ S. ROJAS R.*
}

\section{RESUMEN}

En las Estaciones Experimentales del Instituto de Investigaciones Agropecuarias de Chile (INIA), en Santiago (La Platina) y Osorno (Remehue), se establecieron dos ensayos de evaluación, por dos temporadas consecutivas con el propósito de evaluar el comportamiento de variedades y líneas experimentales de diverso origen, introducidas por el Centro Internacional de la Papa, (CIP). Para ambas temporadas se incluyeron 19 genotipos de papa, de los cuales 15 correspondieron a cultivares introducidos. Como testigo se utilizaron las variedades comerciales: Yagana-INIA, Ultimus, Mirka y Desiree. De las evaluaciones efectuadas durante dos años, el cultivar más promisorio fue Serrana-INTA, con un rendimiento comercial promedio para Osorno y Santiago, de 63.8 y 45.8 t/ha, respectivamente. El cultivar Piratini, también presentó un amplio nivel de adaptación, y mantuvo un comportamiento uniforme en ambas Estaciones Experimentales.

Palabras Claves Adicionales: Variedades, CIP, Chile, genotipos.

\section{ABSTRACT}

\section{Potato Genotypcs Performance in Chile}

Two evaluation assays were established at Experimental Stations of the Agricultural Research Institute of Chile, INIA, in Santiago and Osorno, during two years. The purpose was to evaluate the varieties and experimental lines performance of different origin, introduced from the International Potato Center (CIP). In both years were included 19 potato genotipes, from which 15 were introduced varieties. The commercial varieties Yagana-INIA, Ultimus, Mirka and Desiree, were used as checks. Serrana-INTA, was the best during two years, with an average commercial yield, in Osorno and Santiago, of 63.8 and 45.8 t/ha, respectively. Paratiní cultivar appears with a great range of adaptation, having a uniform performance in both experimental stations.

Additional Index Words: Varieties, CIP, Chile, genotypes.

El Proyecto de Fitomejoramiento de Papa del Instituto de Investigaciones Agropecuarias (INIA) de Chile, tiene como objetivo principal desarrollar e incorporar al cultivo comercial nuevas variedades, con características de alto

Ingenieros Agrónomos, Programa de Papa. Estación Experimental Remehue, Instituto de Investigaciones Agropecuarias, INIA. Casilla 24 - O, Osorno, Chile. 
rendimiento, amplia adaptación, buena calidad culinaria, y ser resistentes o tolerantes a las principales enfermedades y plagas que afecten al cultivo en el país. Para lograr este fin, los países en vías de desarrollo pueden optar por una serie de mecanismos, tales como la introducción de cultivares y materiales genéticos con posibilidades de adaptación a las condiciones agroclimáticas del país o bien desarrollar un programa de cruzamientos e hibridaciones, con el objeto de obtener variedades nacionales (2). El Programa de Papa del INIA, ha concentrado todo su esfuerzo y recursos en el desarrollo de esta segunda alternativa.

La introducción de material genético de otros países no se ha descartado, puesto que representa un papel importante en un programa de mejoramiento y permite la acumulación de factores genéticos tales como: alto rendimiento, adaptación, calidad y resistencia a plagas y enfermedades que se encuentran dispersos en el material introducido (3). Estos factores pueden transferirse al nuevo material que se está creando a través de cruzamientos. Del Centro Internacional de la Papa (CIP), se ha introducido una serie de variedades y líneas experimentales de diverso origen, con el propósito de utilizarlas como progenitores dentro del programa de cruzamientos.

Las condiciones de día largo, bajo las cuales crece el cultivo de papa en Chile, difieren de algunas de las zonas de origen de los cultivares introducidos. Por esta razón, como una manera de evaluar el rendimiento y adaptación de estos genotipos bajo las condiciones chilenas, se establecieron dos ensayos ubicados en dos localidades del país por dos temporadas consecutivas. El objetivo final fue seleccionar aquellas líneas y variedades de mejor comportamiento.

\section{MATERIALES Y MÉTODOS}

Los ensayos de evaluación se establecieron en las Estaciones Experimentales del Instituto de Investigaciones Agropecuarias (INIA), en Santiago $33^{\circ}-6^{\prime}$ latitud sur y Osorno $40^{\circ}-5^{\prime}$ latitud sur.

En ambas temporadas se evaluaron 19 genotipos de papa, de los cuales 15 correspondieron a variedades y líneas experimentales introducidas del CIP (Tabla 1). Como testigo se utilizaron cuatro cultivares comerciales bajo certificación en el país: Yagana INIA, Ultimus, Mirka y Desiree.

El diseño experimental utilizado estuvo conformado por bloques al azar con tres repeticiones. Cada parcela constó de dos surcos de $7.5 \mathrm{~m}$ de largo, separados a $0.75 \mathrm{~m}$ cada uno, lo cual equivale a una superficie de $11.20 \mathrm{~m}^{2}$ por tratamiento y repetición. 
TABLA 1. Genotipos introducidos del Centro Internacional de la Papa CIP, evaluados durante dos temporadas en las localidades de Santiago y Osorno (Chile).

\begin{tabular}{|l|c|}
\hline $\begin{array}{l}\text { Líneas y } \\
\text { variedades }\end{array}$ & $\begin{array}{c}\text { País de } \\
\text { origen }\end{array}$ \\
\hline Serrana— INTA & Argentina \\
\hline B 71240.2— INTA & Argentina \\
\hline Piratiní-EMBRAPA & Brasil \\
\hline Huinkul & Argentina \\
\hline 1 -1035 & India \\
\hline Santo Amor-EMBRAPA & Brasil \\
\hline Atzimba— INIA & México \\
\hline Wauseon & USA \\
\hline Loman & Holanda \\
\hline Montsana—INIA & México \\
\hline Capiro- ICA & Colombia \\
\hline Rosita- INIA & México \\
\hline Pentland Dell & Gran Bretaña \\
\hline Pentland Crown & Gran Bretaña \\
\hline Roslin Castle & Gran Bretaña \\
\hline
\end{tabular}

Durante el desarrollo del ensayo se tomaron datos de emergencia, vigor y precocidad. La cosecha se realizó a los 150 días de la siembra, y el rendimiento se evaluó en tres tamaños: menos de $35 \mathrm{~mm}, 35$ a $65 \mathrm{~mm}$, y más de $65 \mathrm{~mm}$, de diámetro, que corresponden a cohillo, semilla y consumo, respectivamente. A partir de estos datos se calculó el rendimiento comercial, determinado por los tubérculos mayores de $35 \mathrm{~mm}$ de diámetro. A los resultados obtenidos se les hizo un análisis de varianza y separación de promedios por la prueba de Duncan, con una probabilidad de error de tipo I 0.05- Luego se calculó el índice de comportamiento para rendimiento comercial y el coeficiente de diferenciación del ensayo.

\section{RESULTADOS Y DISCUSIÓN}

Los resultados de las temporadas 1985-86 y 1986-87 se presentan en las Tablas 2 y 3 para ambas localidades.

Los rendimientos observados en Osorno son mayores, en relación con los obtenidos en Santiago, lo cual podría explicarse principalmente por las excelentes condiciones agroclimáticas existentes en la zona sur de Chile, que favorecen el desarrollo del cultivo de papa. 
TABLA 2. Rendimiento comercial en toneladas por hectárea e índice de comportamiento en porcentaje, de variedades y líneas experimentales introducidas del CIP. Estación Experimental Renehue, Osorno (Chile).

\begin{tabular}{|c|c|c|c|c|c|c|}
\hline \multirow[t]{2}{*}{ Variedades } & \multicolumn{3}{|c|}{$\begin{array}{c}\text { Rendimiento } \\
\text { Comercial }\end{array}$} & \multicolumn{3}{|c|}{$\begin{array}{c}\text { Indice de } \\
\text { Comportamiento }\end{array}$} \\
\hline & 1985-86 & 1986-87 & $\bar{X}$ & 1985-86 & 1986-87 & $\bar{X}$ \\
\hline Serrana & 77.5 & 50.1 & 63.8 & 88.9 & 68.8 & 78.9 \\
\hline В 71240.2 & --- & 47.9 & 47.9 & & 43.8 & 43.8 \\
\hline Piratiní & 66.1 & 47.5 & 56.8 & 5.6 & 37.5 & 21.6 \\
\hline Yagana INIA & 72.6 & 44.8 & 58.7 & 61.1 & 37.5 & 49.3 \\
\hline Ultimus & 53.5 & 43.7 & 48.6 & 0.0 & 31.8 & 15.7 \\
\hline Mirka & 56.3 & 42.9 & 49.6 & 0.0 & 31.3 & 15.7 \\
\hline Huinkul & 57.7 & 41.9 & 49.8 & 0.0 & 18.8 & 9.4 \\
\hline I - 1035 & 57.6 & 41.8 & 49.7 & 0.0 & 18.8 & 9.4 \\
\hline Desiree & 58.1 & 41.3 & 49.7 & 0.0 & 18.8 & 6.3 \\
\hline Santo Amor & 64.7 & 40.0 & 52.4 & 0.0 & 13.5 & 3.2 \\
\hline Atzimba & 73.0 & 39.3 & 56.2 & 61.1 & 6.3 & 30.6 \\
\hline Wauseon & 55.8 & 36.2 & 46.0 & 0.0 & 0.0 & 0.0 \\
\hline Loman & 63.6 & 34.3 & 49.0 & 0.0 & 0.0 & 0.0 \\
\hline Montsana & 57.6 & 34.0 & 45.8 & 0.0 & 0.0 & 0.0 \\
\hline Capiro & 58.4 & 33.5 & 46.0 & 0.0 & 0.0 & 0.0 \\
\hline Rosita & 60.3 & 32.9 & 46.6 & 0.0 & 0.0 & 0.0 \\
\hline Pentland Dell & 61.7 & 31.2 & 46.5 & 0.0 & 0.0 & 0.0 \\
\hline Pentland Crown & 65.1 & -- & 65.1 & 0.0 & --- & 0.0 \\
\hline Roslin Castle & 58.4 & --- & 58.4 & 0.0 & --- & 0.0 \\
\hline
\end{tabular}


TABLA 3. Rendimiento comercial en toneladas por hectárea e índice de comportamiento en porcentaje, variedades y líneas experimentales introducidas del CIP. Estación Experimental La Platina, Stgo. (Chile).

\begin{tabular}{|c|c|c|c|c|c|c|}
\hline \multirow[t]{2}{*}{ Variedades } & \multicolumn{3}{|c|}{$\begin{array}{c}\text { Rendimiento } \\
\text { Comercial }\end{array}$} & \multicolumn{3}{|c|}{$\begin{array}{c}\text { Indice de } \\
\text { Comportamiento }\end{array}$} \\
\hline & 1985-86 & 1986-87 & $\bar{X}$ & 1985-86 & $1986-87$ & $\bar{X}$ \\
\hline Serrana & 58.1 & 33.4 & 45.8 & 100.0 & 93.3 & 96.6 \\
\hline Piratiní & 51.1 & 27.9 & 39.5 & 94. & 53.3 & 73.7 \\
\hline Yagana INIA & 40.1 & 18.9 & 29.5 & 23.5 & 6.7 & 15.1 \\
\hline Ultimus & 45.8 & 25.2 & 35.5 & 47.1 & 26.7 & 36.9 \\
\hline Mirka & 45.5 & 22.8 & 34.2 & 47.1 & 6.7 & 26.9 \\
\hline Huinkul & 36.0 & 31.8 & 33.7 & 5.9 & 80.0 & 43.0 \\
\hline I - 1035 & 43.1 & 30.0 & 36.6 & 29.4 & 73.3 & 51.4 \\
\hline Desiree & 42.2 & 24.6 & 33.4 & 23.5 & 20.0 & 21.8 \\
\hline Santo Amor & 43.8 & 23.4 & 33.6 & 35.5 & 6.7 & 21.0 \\
\hline Atzimba & 35.8 & 24.1 & 30.0 & 5.9 & 20.0 & 13.0 \\
\hline Wauseon & 29.9 & 16.8 & 23.4 & 5.9 & 0.0 & 3.0 \\
\hline Loman & 44.5 & 26.7 & 36.6 & 41.2 & 40.0 & 40.6 \\
\hline Montsana & 41.4 & 30.5 & 36.0 & 23.5 & 80.0 & 51.8 \\
\hline Capiro & 28.9 & 25.9 & 22.4 & 0.0 & 33.3 & 16.7 \\
\hline Rosita & 39.9 & 21.6 & 30.8 & 23.5 & 6.7 & 15.1 \\
\hline Penthland Dell & 42.4 & 25.9 & 34.2 & 23.5 & 33.3 & 28.4 \\
\hline Pentland Crown & 39.2 & --- & 39.2 & 11.8 & --- & 11.8 \\
\hline Roslin Castle & 45.5 & --- & 45.5 & 47.1 & --- & 47.1 \\
\hline
\end{tabular}




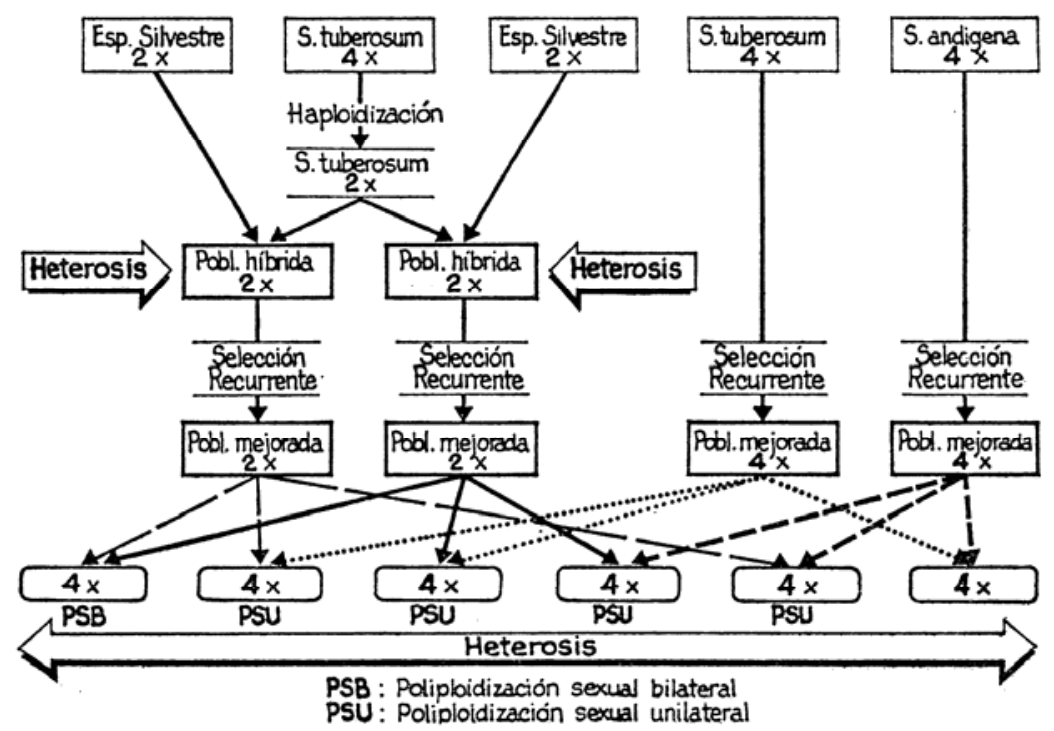

FIGURA 1. Esquema de mejoramiento genético de la papa mediante manipulaciones de ploidía.

De los cultivares evaluados el más promisorio fue Serrana INTA, con un rendimiento comercial promedio para Osorno y Santiago de 63.8 y 45.8 t/ha., respectivamente. En la zona sur (Remehue), le siguen en productividad las variedades Yagana-INIA (testigo), Piratiní y Atzimba. Por otro lado, en Santiago el cultivar Yagana-INIA pierde productividad destacándose en esta zona la línea, India-1035.

Al comparar los rendimientos para dos temporadas dentro de una misma localidad, se observa claramente una disminución de la productividad durante 1986-87 con respecto a 1985-86, en Osorno. Esto se puede explicar fundamentalmente debido a la severa sequía que afectó a la zona sur de Chile durante los meses de diciembre, enero y febrero, período durante el cual se produce el máximo crecimiento de las plantas. Lo anterior se tradujo en un mal comportamiento y productividad de los cultivares Atzimba, Piratiní y Pentland Dell, y que al parecer presentan una mayor sensibilidad a períodos prolongados de stress hídrico.

Por otra parte, en la Estación Experimental La Platina (Santiago), se observó también una importante disminución de los rendimientos para la segunda temporada de evaluación. La razón fue básicamente fitosanitaria, debido a que las plantas se vieron expuestas a un fuerte ataque de Colletotrichum atramentarium. La antracnosis causada por este patógeno es una enfermedad de reciente aparición en el país, que en general afecta a las variedades de mayor precocidad (1). En el ensayo, los cultivares más afectados por este hongo fueron Wauseon, Santo Amor y Ultimus.

En la Tabla 4 se muestra un resumen de los resultados obtenidos para las dos temporadas en ambas localidades. En efecto, la variedad que produjo los mejores rendimientos fue Serrana INTA, pues presentó la mayor producción comercial promedio tanto para Santiago como para 
Osorno. Otro cultivar de amplio nivel de adaptación es Piratiní, que mantiene también un comportamiento uniforme en ambas localidades de Chile.

TABLA 4. Rendimiento comercial promedio en toneladas por hectárea en dos temporadas (1986 y 1987), de evaluación de variedades y líneas experimentales, introducidas del Centro Internacional de la Papa.

\begin{tabular}{lcccc}
\hline Líneas y variedades & \multicolumn{2}{c}{ Santiago $\left(33^{\circ} 6^{\prime}\right.$ L.S.) } & \multicolumn{2}{c}{ Osorno $\left(40^{\circ} 5^{\prime}\right.$ L.S.) } \\
\hline Serrana & 45.8 & $(1)$ & 63.8 & $(1)$ \\
Piratiní & 39.5 & $(2)$ & 56.8 & $(2)$ \\
Ultimus & 35.6 & $(6)$ & 48.1 & $(\mathrm{U})$ \\
Mirka & 34.1 & $(8)$ & 49.6 & $(9)$ \\
Loman & 35.6 & $(5)$ & 49.0 & $(10)$ \\
Santo Amor & 33.6 & $(9)$ & 52.4 & $(5)$ \\
I - 1035 & 36.6 & $(3)$ & $49-7$ & $(7)$ \\
Pentland Dell & 34.2 & $(7)$ & 46.5 & $(13)$ \\
Desiree & 33.4 & $(\mathrm{U})$ & 49.7 & $(8)$ \\
Montsana & 35.9 & $(4)$ & 45.8 & $(16)$ \\
Yagana & 29.5 & $(14)$ & 58.7 & $(2)$ \\
Rosita & $30-8$ & $(12)$ & 46.6 & $(12)$ \\
Huinkul & 33.6 & $(10)$ & 45.8 & $(6)$ \\
Atzimba & 29.9 & $(13)$ & 56.2 & $(4)$ \\
Wauseon & 23.3 & $(16)$ & 46.0 & $(14)$ \\
Capiro & 27.4 & $(15)$ & 46.0 & $(15)$ \\
Promedio & 33.7 & & 50.9 & \\
\hline
\end{tabular}

\section{REFERENCIAS BIBLIOGRÁFICAS}

1. Fernández, C. 1987. Reacción de cultivares de papa al daño causado por Colletotrichum atramentarium, Instituto de Investigaciones Agropecuarias Osorno, Chile. (VII Informe Anual Proyecto Fitomejoramiento de papa).

2. Kalazich, J. 1983. Cómo se obtiene una nueva variedad de papa. Instituto de Investigaciones Agropecuarias, Osorno, Chile (Boletín Técnico $\mathrm{N}^{\circ} 63$ ).

3. Sandoval, C. 1986. Base genética del Proyecto Fitomejoramiento de la papa del INIA y etapas dentro del proceso de creación de una nueva variedad. Instituto de Investigaciones Agropecuarias Osorno, Chile. (Segundo Curso Internacional de Producción y Almacenamiento de Papa Semilla Certificada) 